\title{
Transmittance Magneto-Optical Responses of One-Dimensional Magnetophotonic Heterostructures
}

\author{
R. ABDi-GHALEH ${ }^{a, *}$ AND M. AsAD ${ }^{b}$ \\ ${ }^{a}$ Department of Laser and Optical Engineering, University of Bonab, 5551761167, Bonab, Iran \\ ${ }^{b}$ School of Electrical and Computer Engineering, Shiraz University, 71345-1585 Shiraz, Iran \\ (Received December 5, 2013; in final form March 24, 2014)
}

\begin{abstract}
Transmittance magneto-optical responses of one-dimensional magnetophotonic heterostructures are investigated using 4 by 4 transfer matrix method. It is shown that in a simple magnetophotonic heterostructure constituted of two different periodic structures, the enhanced Faraday rotation associated with high transmittance at a desired wavelength can be realized with specifying optimized repetition numbers and adjusting the thicknesses of the substructure layers. However, the unique features of multichanneled enhanced Faraday rotations are obtained for different configurations of a multiple magnetophotonic heterostructure and the most suitable one to simultaneously support the both resonance wavelengths of $1.3 \mu \mathrm{m}$ and $1.55 \mu \mathrm{m}$ are introduced. These wavelengths are currently used in telecommunication systems. Then the obtained results may have potential applications in designing the multi-function single magneto-optical devices such as multiple Faraday rotators and wavelength division multiplexing systems.
\end{abstract}

DOI: 10.12693/APhysPolA.126.705

PACS: 42.70.Qs, 75.70.Cn, 85.70.Sq

\section{Introduction}

Photonic crystals (PCs) are periodic dielectric structures that are proposed to control and manipulate the propagation of electromagnetic (EM) waves $[1,2]$. After the pioneering papers of Yablonovitch and John in 1987 $[3,4]$ exponential growth of theoretical and experimental researches on PCs have been started and their potential applications continue to be examined. Appearing of the photonic band gap (PBG) where the EM waves with frequencies within the gap cannot propagate through the structure, is the main characteristic of the PCs. Periodic one-dimensional PCs (1D-PCs) are composed of an ordered sequence of two different dielectric slabs. One of the most interesting aspects of $1 \mathrm{D}-\mathrm{PCs}$ is related to presence of a defect layer in the periodic structure which gives rises to localization of EM wave and creates a resonance transmittance within the gap, which allows the corresponding EM wave with previously forbidden wavelength to propagate inside the structure [4-6].

It has been revealed that the localization of EM waves appears not only in the defective structures, but also in quasiperiodic systems such as Fibonacci [7] and ThueMorse $[8,9]$ multilayers. Moreover, a kind of the particularly attractive disordered structures are heterostructures which are formed by combination of two or more periodic 1D-PCs with different layer thicknesses or different constituent materials. Distinct PCs have different optical properties, then heterostructures can show many appealing characteristics. For example, extension of the PBG, criterion of omnidirectional reflections and designing of polarization bandpass filters have been studied in number of literatures [10-13].

* corresponding author; e-mail: r.abdi@bonabu.ac.ir
On the other hand, in past several years, considerable attentions have been paid to magnetophotonic crystals (MPCs) due to their capability of providing unique magneto-optical (MO) properties, such as their drastically enhanced Faraday rotations (FRs) [14-17]. The MPCs are formed when the constitutive materials of the PCs are magnetic, or even only a defect layer in the PC is magnetic [16]. The structures with high MO responses are interested to use in many MO devices, such as MO isolators, MO modulators, MO sensors, and MO circulators. Recently, utilizing multicavity MPCs to create multiple passbands inside the PBG, have opened a new window to engineer multi-function single MO-devices which show simultaneously high transmittance and enhanced FRs [18-20].

In this paper, we discuss magnetophotonic heterostructures constituted of dielectric and magnetic multilayers. The MO responses of simple and multiple heterostructures are studied through 4 by 4 transfer matrix method. We show that the enhanced FRs at desire resonance wavelengths in a wide PBG would be obtained considering special design of heterostructures. Such a capability may have potential applications in multi-function single MO-devices.

The outline of our study is as follows: Sect. 2 gives the brief description of 4 by 4 transfer matrix method. In Sect. 3 we have presented our study in two steps. First, for a simple heterostructure consists of two periodic MPCs with different design wavelengths, it is shown that the resonance transmittance would occur at special wavelengths. Then for a limited case of simple heterostructure, the approximated relations to describe the transmittance and FR at the resonance wavelengths are derived analytically. Furthermore, the spectral properties of the simple heterostructure and its substructures are investigated numerically. Second, for different configurations of a multiple heterostructure constituted of four 
periodic MPCs as substructures, the transmittance and FR spectra are studied. Finally, in Sect. 4 the obtained results are summarized.

\section{4 by 4 transfer matrix formalism}

Consider the EM wave propagation through a periodic MPC structure $P_{1}=\{A, B\}^{m}$, where $A$ and $B$ are dielectric and magnetic layers with refractive indexes of $n_{A}$ and $n_{B}$ and optical thicknesses of $n_{A} d_{A}=n_{B} d_{B}=\lambda^{(1)} / 4$. In this representation, $m$ denotes the repetition number and $\lambda^{(1)}$ is design wavelength. It is considered that the whole structure is surrounded by air. To calculate MO responses of magnetic multilayer structures, we use 4 by 4 transfer matrix method and follow the formalism which have been developed by Višňovský [21]. In a medium uniformly magnetized in $z$-axis, the dielectric permittivity of magnetic layer $\hat{\varepsilon}_{B}$ has the following form:

$$
\hat{\varepsilon}_{B}=\left(\begin{array}{ccc}
\varepsilon_{x x} & \varepsilon_{x y} & 0 \\
-\varepsilon_{x y} & \varepsilon_{x x} & 0 \\
0 & 0 & \varepsilon_{z z}
\end{array}\right),
$$

where the nondiagonal term $\varepsilon_{x y}$ corresponds to the magnetic gyration. In linear regime, $\varepsilon_{x y}$ is proportional to the magnetization of the medium and can be tuned by external magnetic field $\boldsymbol{H}_{\text {ext }}$. The dielectric layer is determined by a diagonal tensor $\hat{\varepsilon}_{A}$ as

$$
\hat{\varepsilon}_{A}=\left(\begin{array}{ccc}
\varepsilon_{A} & 0 & 0 \\
0 & \varepsilon_{A} & 0 \\
0 & 0 & \varepsilon_{A}
\end{array}\right) .
$$

The refractive indexes of magnetic and dielectric layers can be defined as $n_{B}=\sqrt{\varepsilon_{x x}}$ and $n_{A}=\sqrt{\varepsilon_{A}}$. For a $J$-layered MPC structure the total transfer matrix for noninteracting left- and right-circularly polarized EM waves

$$
M=\left[D^{(0)}\right]^{-1} \prod_{j=1}^{\mathrm{J}} S^{(j)} D^{(j+1)}
$$

relates the EM field amplitudes of incident and transmitted waves through the characteristic $(S)$ and dynamic $(D)$ matrices. For the case of normal incidence and polarization parallel to multilayer surfaces, the block diagonal $S$ and $D$ matrices are given by

$$
\begin{aligned}
& D^{(j)}=\left[\begin{array}{cccc}
1 & 1 & 0 & 0 \\
N_{+}^{(j)} & -N_{+}^{(j)} & 0 & 0 \\
0 & 0 & 1 & 1 \\
0 & 0 & N_{-}^{(j)} & -N_{-}^{(j)}
\end{array}\right], \\
& S^{(j)}= \\
& {\left[\begin{array}{cccc}
\cos \beta_{+}^{(j)} & \frac{\mathrm{i}}{N_{+}^{(j)}} \sin \beta_{+}^{(j)} & 0 & 0 \\
\mathrm{i} N_{+}^{(j)} \sin \beta_{+}^{(j)} & \cos \beta_{+}^{(j)} & 0 & 0 \\
0 & 0 & \cos \beta_{-}^{(j)} & \frac{\mathrm{i}}{N_{-}^{(j)}} \sin \beta_{-}^{(j)} \\
0 & 0 & \mathrm{i} N_{-}^{(j)} \sin \beta_{-}^{(j)} & \cos \beta_{-}^{(j)}
\end{array}\right] .}
\end{aligned}
$$

Here $N_{ \pm}^{(j)}=\sqrt{\varepsilon_{x x}^{(j)} \pm \mathrm{i} \varepsilon_{x y}^{(j)}}$ represents the complex refractive index for right- and left-circularly polarized EM waves in the $j$-th layer. $\beta_{ \pm}^{(j)}=(2 \pi / \lambda) N_{ \pm}^{(j)} d^{(j)}$ in which $d^{(j)}$ is the thickness of the $j$-th layer and $\lambda$ is the wavelength of incident wave in the vacuum. For a dielectric layer $N_{+}^{(j)}=N_{-}^{(j)}$ and the characteristic and dynamic matrixes consist of identical 2 by 2 sub-blocks. In terms of $M$ matrix components, the transmittance $T(\lambda)$ and Faraday rotation $\Theta_{\mathrm{F}}(\lambda)$ of the MPC can be expressed as follows:

$$
\begin{aligned}
& T(\lambda)=\frac{1}{2}\left(\left|M_{11}\right|^{-2}+\left|M_{33}\right|^{-2}\right), \\
& \Theta_{\mathrm{F}}(\lambda)=-\frac{1}{2} \arg \left(\frac{M_{11}}{M_{33}}\right) .
\end{aligned}
$$

\section{MO responses of magnetophonic heterostructures}

\subsection{A simple magnetophotonic heterostructure}

In order to study the MO responses of magnetophotonic heterostructures, at first, we introduce a simple heterostructure with two substructures. Defining the left substructure as $P_{\mathrm{L}}=\left\{A_{1}, B_{1}\right\}^{m_{1}}$ with the optical thicknesses of $n_{A} d_{A_{1}}=n_{B} d_{B_{1}}=\lambda_{\mathrm{L}} / 4$ and the right substructure as $P_{\mathrm{R}}=\left\{B_{2}, A_{2}\right\}^{m_{2}}$ with the optical thicknesses of $n_{A} d_{A_{2}}=n_{B} d_{B_{2}}=\lambda_{\mathrm{R}} / 4$, we construct a simple-heterostructure $\mathrm{SH}$ as

$$
\begin{aligned}
S H & =P_{\mathrm{L}} U P_{\mathrm{R}}=\left\{A_{1}, B_{1}\right\}^{m_{1}}\left\{B_{2}, A_{2}\right\}^{m_{2}} \\
& =\{\underbrace{A_{1}, B_{1}, \ldots, A_{1}, B_{1}}_{m_{1} \text { pair of } A_{1}, B_{1}} \mid \underbrace{B_{2}, A_{2}, \ldots, B_{2}, A_{2}}_{m_{2} \text { pair of } B_{2}, A_{2}}\},
\end{aligned}
$$

in which $\lambda_{\mathrm{L}}$ and $\lambda_{\mathrm{R}}$ are the design wavelengths of left and right substructures, respectively. The $\mathrm{SH}$ has $2\left(m_{1}+m_{2}\right)$ layers and is surrounded by air. The left and right substructures consist of identical dielectric (A) and magnetic materials (B), but with different thicknesses. We utilize $\mathrm{SiO}_{2}$ and cerium substituted yttrium iron garnet ( Ce:YIG) as the dielectric and magnetic layers. Ce:YIG is used because it turns out to be one of the most attractive materials for practical applications due to low absorption in infrared region and large MO responses. The dielectric permittivity of $\mathrm{SiO}_{2}$ is $\varepsilon_{A}=2.19$ and the magnetic Ce:YIG layer has dielectric tensor elements $\varepsilon_{x x}=$ 4.884 and $\varepsilon_{x y}=0.009 \mathrm{i}$ at telecommunication wavelength $\lambda=1.55 \mu \mathrm{m}$ [14]. As it is understood from the dielectric constants, in the infrared region of electromagnetic spectrum these materials can be considered lossless, with a very good approximation. The corresponding refractive indexes are $n_{A}=n_{\mathrm{SiO}_{2}}=1.48$ and $n_{B}=n_{\mathrm{Ce}: \mathrm{YIG}}=2.21$. The specific FR of a single layer Ce:YIG can be calculated by $\theta_{\mathrm{F}}=\frac{\pi}{\lambda} \Delta n \cong-0.47 \mathrm{deg} / \mu \mathrm{m}$ at $\lambda=1.55 \mu \mathrm{m}[22]$. Here $\Delta n$ is the difference of the refractive indexes of leftand right- circularly polarized EM waves in the magnetic layer.

According to Eq. (3), the total transfer matrix for the heterostructure SH can be represented by Eq. (9): 


$$
\begin{aligned}
& M_{1}=\left\{\left[D^{(0)}\right]^{-1} S^{\left(A_{1}\right)} S^{\left(B_{1}\right)} \ldots S^{\left(A_{1}\right)} S^{\left(B_{1}\right)}\right. \\
& \times D^{\left(2 m_{1}+1\right)} \mid\left[D^{\left(2 m_{1}+1\right)}\right]^{-1} S^{\left(B_{2}\right)} S^{\left(A_{1}\right)} \\
& \left.\quad \ldots S^{\left(B_{2}\right)} S^{\left(A_{1}\right)}\left[D^{\left(2\left(m_{1}+m_{2}\right)+1\right)}\right]^{-1}\right\} .
\end{aligned}
$$

face of the left and right substructures

$$
D^{\left(2 m_{1}+1\right)}\left[D^{\left(2 m_{1}+1\right)}\right]^{-1}=I,
$$

which $I$ is the unit matrix. Then the central part of the $M_{1}$ is simplified as the product of $S^{\left(B_{1}\right)}$ and $S^{\left(B_{2}\right)}$, as shown in Eq. (11):

Looking the center of $M_{1}$, it is obvious that at the inter-

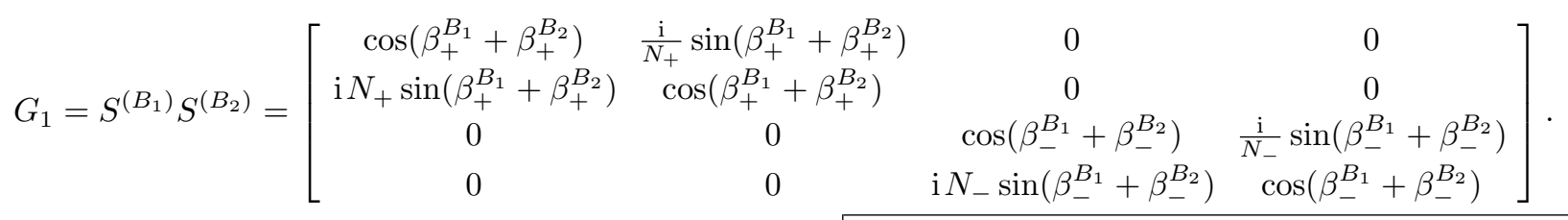

The argument of the trigonometric functions can be written as

$$
\begin{gathered}
\beta_{ \pm}^{B_{1}}+\beta_{ \pm}^{B_{2}}=\frac{2 \pi}{\lambda} N_{ \pm}^{B}\left(d_{B_{1}}+d_{B_{2}}\right) \\
=\frac{\pi}{2} \frac{N_{ \pm}^{B}}{n_{B}}\left(\frac{\lambda_{\mathrm{L}}+\lambda_{\mathrm{R}}}{\lambda}\right) .
\end{gathered}
$$

At this step, it is illustrative to study the case that $\varepsilon_{x y}=0$; which may be obtained by setting the external magnetic field $\boldsymbol{H}_{\text {ext }}$ to zero. Then for all magnetic layers $N_{+}^{B}=N_{-}^{B}=n_{B}$ and the aforementioned equation will be reduced to

$$
\beta_{ \pm}^{B_{1}}+\beta_{ \pm}^{B_{2}}=\frac{\pi}{2}\left(\frac{\lambda_{\mathrm{L}}+\lambda_{\mathrm{R}}}{\lambda}\right)
$$

We set the wavelength of incident EM wave as

$$
\lambda_{q}=\left(\lambda_{\mathrm{L}}+\lambda_{\mathrm{R}}\right) / 2 q,
$$

where $q$ is an integer. We call this wavelength the resonance wavelength of the heterostructure. So, the argument of trigonometric functions of Eq. (11) will be $q \pi$ and we have

$$
G_{1}=(-1)^{q} I .
$$

Considering Eq. (9), again the central part of $M_{1}$ is simplified to $G_{2}=(-1)^{q} S^{\left(A_{1}\right)} S^{\left(A_{2}\right)}$. Product of the characteristic matrices of $S^{\left(A_{1}\right)}$ and $S^{\left(A_{2}\right)}$ under the condition of Eq. (14) gives a unit matrix multiplied by $(-1)^{q}$. Repeating this procedure, it can be concluded that when the left and right substructures of SH have identical repetition numbers $m_{1}=m_{2}=m$, the total transfer matrix for the heterostructure $\mathrm{SH}$ is

$$
M_{1}=(-1)^{2 m q} I \text {. }
$$

Using Eqs. $(6,7)$, the transmittance and FR of the $\mathrm{SH}$ are

$$
T=1, \quad \Theta_{\mathrm{F}}=0 .
$$

As a result, for the case of $\varepsilon_{x y}=0$, EM waves with the resonant wavelengths of $\lambda_{q}=\left(\lambda_{\mathrm{L}}+\lambda_{\mathrm{R}}\right) / 2 q$ would be perfectly transmitted through the heterostructure $\mathrm{SH}$ without any FR.

Now we come back to the general case $\varepsilon_{x y} \neq 0$. For most magnetic materials the nondiagonal component of dielectric permittivity tensor is much smaller than the diagonal one. For Ce:YIG we can consider $\varepsilon_{x y}=\mathrm{i} g$, then $g / \varepsilon_{x x}$ will be of the order of $10^{-3}$ and Eq. (12) can be approximated by

$$
\beta_{ \pm}^{B_{1}}+\beta_{ \pm}^{B_{2}} \approx \frac{\pi}{2}(1 \mp \delta)\left(\frac{\lambda_{\mathrm{L}}+\lambda_{\mathrm{R}}}{\lambda}\right), \delta=\frac{g}{2 \varepsilon_{x x}} \ll 1 .
$$

Setting the wavelength of incident wave as Eq. (14), we have

$$
\beta_{ \pm}^{B_{1}}+\beta_{ \pm}^{B_{2}} \approx q \pi(1 \mp \delta)
$$

The simplification of $G_{1}$ (Eq. (11)) can be made through the first order approximation of trigonometric functions regarding the resonant wavelengths i.e. Eq. (19):

$$
\begin{aligned}
& G_{1}=S^{\left(B_{1}\right)} S^{\left(B_{2}\right)}=(-1)^{q} \\
& \times\left[\begin{array}{cccc}
1 & -\frac{\mathrm{i}}{N_{+}^{B}}(q \pi \delta) & 0 & 0 \\
-\mathrm{i} N_{+}^{B}(q \pi \delta) & 1 & 0 & 0 \\
0 & 0 & 1 & \frac{\mathrm{i}}{N_{-}^{B}}(q \pi \delta) \\
0 & 0 & \mathrm{i} N_{-}^{B}(q \pi \delta) & 1
\end{array}\right] .
\end{aligned}
$$

Comparing with Eq. (15), it can be seen that the nondiagonal components in Eq. (20) originate from the magnetic gyration $g$.

Taking into account that for dielectric layers $A_{1}$ and $A_{2}$,

$$
\begin{aligned}
& \beta_{+}^{\mathrm{A}_{1}}=\beta_{-}^{\mathrm{A}_{1}}=\beta^{\mathrm{A}_{1}}=q \pi\left(\frac{\lambda_{\mathrm{L}}}{\lambda_{\mathrm{L}}+\lambda_{\mathrm{R}}}\right), \\
& \beta_{+}^{\mathrm{A}_{2}}=\beta_{-}^{\mathrm{A}_{2}}=\beta^{\mathrm{A}_{2}}=q \pi\left(\frac{\lambda_{\mathrm{R}}}{\lambda_{\mathrm{L}}+\lambda_{\mathrm{R}}}\right) .
\end{aligned}
$$

Then $\beta^{\mathrm{A}_{1}}+\beta^{\mathrm{A}_{2}}=q \pi$ and we define the parameter $\Delta \beta^{A}$ as

$$
\begin{aligned}
& \Delta \beta^{A}=\beta^{\mathrm{A}_{2}}-\beta^{\mathrm{A}_{1}}=q \pi\left(\frac{\Delta \lambda}{\lambda_{\mathrm{L}}+\lambda_{\mathrm{R}}}\right), \\
& \Delta \lambda=\lambda_{\mathrm{R}}-\lambda_{\mathrm{L}} .
\end{aligned}
$$

Considering the central part of $M_{1}$ we can calculate $G_{2}$ matrix as 


$$
G_{2}=S^{\left(A_{1}\right)} G_{1} S^{\left(A_{2}\right)}=(-1)^{q}\left[\begin{array}{cccc}
G_{2}^{11} & G_{2}^{12} & 0 & 0 \\
G_{2}^{21} & G_{2}^{22} & 0 & 0 \\
0 & 0 & G_{2}^{33} & G_{2}^{34} \\
0 & 0 & G_{2}^{43} & G_{2}^{44}
\end{array}\right]
$$

where

$$
\begin{aligned}
& G_{2}^{11}=Z_{+}, \quad G_{2}^{12}=U_{+}, \quad G_{2}^{21}=V_{+}, \quad G_{2}^{22}=Y_{+}, \\
& G_{2}^{33}=Z_{-}, \quad G_{2}^{34}=U_{-}, \quad G_{2}^{43}=V_{-}, \quad G_{2}^{44}=Y_{-},
\end{aligned}
$$

in which

$$
\begin{aligned}
Z_{ \pm} & =(-1)^{q}+\frac{q \pi \delta}{2} \sin \left(\Delta \beta^{A}\right)\left(\mp \frac{N_{ \pm}^{B}}{n_{A}} \pm \frac{n_{A}}{N_{ \pm}^{B}}\right), \\
Y_{ \pm} & =(-1)^{q}+\frac{q \pi \delta}{2} \sin \left(\Delta \beta^{A}\right)\left( \pm \frac{N_{ \pm}^{B}}{n_{A}} \mp \frac{n_{A}}{N_{ \pm}^{B}}\right), \\
U_{ \pm} & =\frac{\mathrm{i} q \pi \delta}{2}\left[\cos \left(\Delta \beta^{A}\right)\left( \pm \frac{N_{ \pm}^{B}}{n_{A}^{2}} \mp \frac{1}{N_{ \pm}^{B}}\right)\right. \\
& \left.\mp(-1)^{q}\left(\frac{N_{ \pm}^{B}}{n_{A}^{2}}+\frac{1}{N_{ \pm}^{B}}\right)\right], \\
V \pm & =\frac{\mathrm{i} q \pi \delta}{2}\left[\cos \left(\Delta \beta^{A}\right)\left( \pm \frac{n_{A}^{2}}{N_{ \pm}^{B}} \mp N_{ \pm}^{B}\right)\right. \\
& \left.\mp(-1)^{q}\left(\frac{n_{A}^{2}}{N_{ \pm}^{B}}+N_{ \pm}^{B}\right)\right] .
\end{aligned}
$$

For the heterostructure SH with $m_{1}=m_{2}=1$ the total transfer matrix can be written as

$$
\left.M_{1}\right|_{m_{1}=m_{2}=1}=\left[D^{(\text {air })}\right]^{-1} G_{2} D^{(\text {air })} .
$$

After a straightforward derivation, the key components $M_{1}^{11}$ and $M_{1}^{33}$ can be obtained as

$$
\begin{aligned}
& M_{1}^{11}=\frac{(-1)^{q}}{2}\left(2(-1)^{q}+\frac{\mathrm{i} q \pi \delta}{2} \gamma_{+}\right), \\
& M_{1}^{33}=\frac{(-1)^{q}}{2}\left(2(-1)^{q}+\frac{\mathrm{i} q \pi \delta}{2} \gamma_{-}\right),
\end{aligned}
$$

where

$$
\gamma_{ \pm}=\frac{ \pm 1}{N_{ \pm}^{B}} \sigma_{ \pm},
$$

with

$$
\begin{aligned}
\sigma_{ \pm} & =\left[\left(N_{ \pm}^{B}\right)^{2}+n_{A}^{2}\right]\left(1+\frac{1}{n_{A}^{2}}\right) \\
& \times\left(\cos \left(\Delta \beta^{A}\right)+(-1)^{q}\right)-2 \cos \left(\Delta \beta^{A}\right) .
\end{aligned}
$$

It is clear that the $\gamma_{ \pm}$is a real parameter with the order of unit, then $\delta \gamma_{ \pm} \ll 1$ and $\left|M_{1}^{11}\right|^{-2}$ and $\left|M_{1}^{33}\right|^{-2}$ can be approximated as

$$
\left|M_{1}^{11}\right|^{-2} \approx 1-\left(\frac{q \pi \delta}{4} \gamma_{+}\right)^{2},
$$

$$
\left|M_{1}^{33}\right|^{-2} \approx 1-\left(\frac{q \pi \delta}{4} \gamma_{-}\right)^{2} .
$$

Finally, the transmittance and $\mathrm{FR}$ of the $S H_{1}=$ $\left\{A_{1}, B_{1}\right\}^{1}\left\{B_{2}, A_{2}\right\}^{1}$ is

$$
\begin{aligned}
& T \approx 1-\frac{(q \pi \delta)^{2}}{32}\left(\gamma_{+}^{2}+\gamma_{-}^{2}\right), \\
& \Theta_{\mathrm{F}} \approx-\frac{(-1)^{q} q \pi \delta}{8}\left(\gamma_{-}-\gamma_{+}\right) .
\end{aligned}
$$

Comparing the achieved transmittance and FR with the case of nonmagnetic heterostructure i.e. Eq. (17), we see that the transmittance at resonant wavelength of heterostructure has been slightly violated from the perfect value and a small FR has appeared. The slight violations of transmittance and the values of FR are dependent on the $\delta$ by second and first power orders, respectively. However, the parameter $\delta\left(=g / 2 \varepsilon_{x x}\right)$ is directly related to the magnetic gyration $g$, while the $\gamma_{ \pm}$is very weakly affected by small variations of the $g$ (see Eq. (29)). Consequently, knowing that $\delta<1$, the slight decrease of the $g$ will cause the faster increase of FR value and slower diminishing of perfect transmittance violations. From Eq. (32), it can be clearly seen that for the case $g \rightarrow 0$, the same results of nonmagnetic heterostructure will be obtained, i.e. Eq. (17).

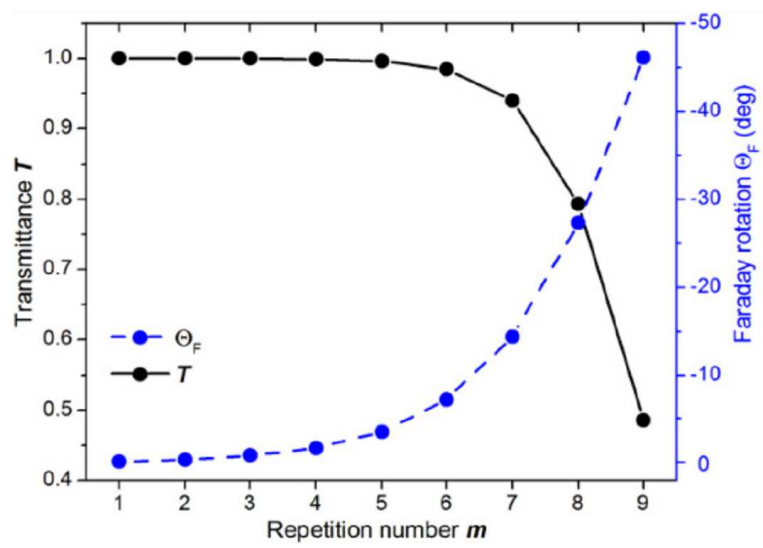

Fig. 1. Transmittance and Faraday rotation for the $\mathrm{SiO}_{2} / \mathrm{Ce}: \mathrm{YIG}$ multilayers as the heterostructure of $\mathrm{SH}=\left\{A_{1}, B_{1}\right\}^{m}\left\{B_{2}, A_{2}\right\}^{m}$ versus the repetition number $m$ at resonance wavelength $\lambda_{1}=1.55 \mu \mathrm{m}$. Here the design wavelengths for left and right substructures are $\lambda_{\mathrm{L}}=1.45 \mu \mathrm{m}$ and $\lambda_{\mathrm{R}}=1.65 \mu \mathrm{m}$.

Actually, finding the explicit analytical relations to express the transmittance and FR at heterostructure resonant wavelength is not straightforward for higher repetition numbers. Hence, we have calculated them numerically using the total transfer matrix of Eq. (9) and final relations of Eqs. $(6,7)$. Figure 1 depicts the transmittance and FR of the SH versus the repetition number $m$. These results are obtained regarding the $\lambda_{\mathrm{L}}=1.45 \mu \mathrm{m}$, $\lambda_{\mathrm{R}}=1.65 \mu \mathrm{m}$ and $q=1$, which corresponds to the heterostructure resonant wavelength of $\lambda_{1}=1.55 \mu \mathrm{m}$. From 
the figure, the trade-off relationship between the transmittance and FR can be seen clearly; for higher repetition numbers, the more enhanced FRs would be obtained with the cost of transmittance. It can be deduced that the heterostructures with repetition numbers of $m=7$ and $m=8$ are appropriate to provide simultaneously high transmittance and enhanced FR. The heterostructure SH shows the FR of $\Theta_{\mathrm{F}}=-14.39 \mathrm{deg}$ and $\Theta_{\mathrm{F}}=-27.35 \mathrm{deg}$ with the transmittance of $T=0.94$ and $T=0.79$, respectively for $m=7$ and $m=8$. These FRs are more than 12 and 20 times larger than the corresponding FRs of a single layer Ce:YIG with a thickness equal to sum of all magnetic layer thicknesses in the $\mathrm{SH}$ with $m=7$ and $m=8$, respectively.

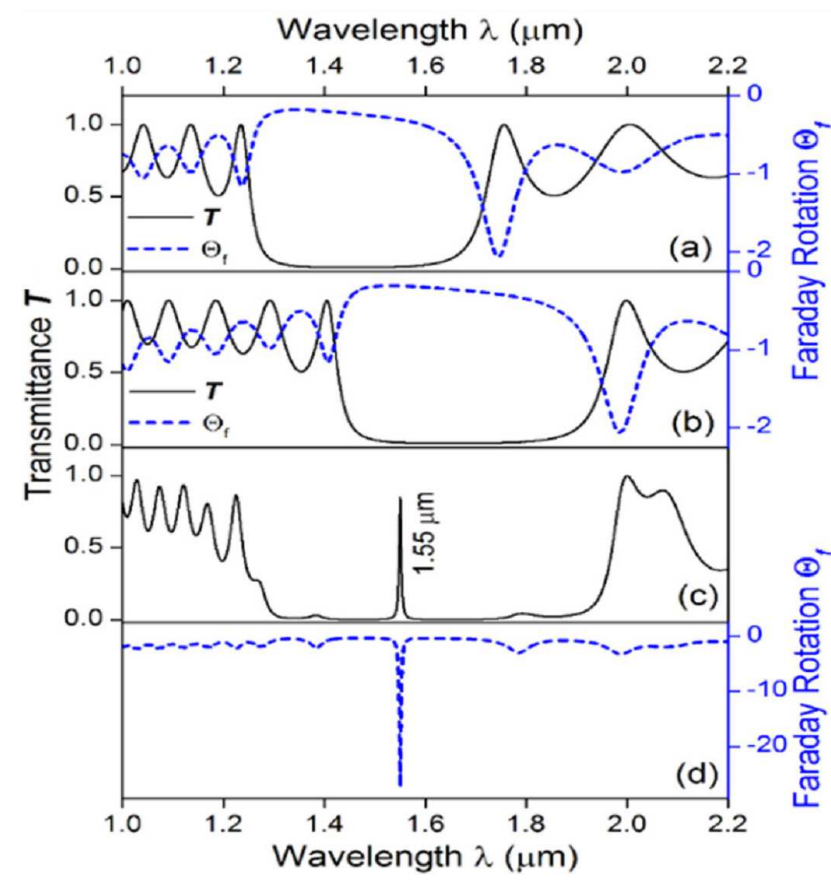

Fig. 2. Transmittance and Faraday rotation spectra for the $\mathrm{SiO}_{2} / \mathrm{Ce}$ :YIG multilayers with the structure as (a) the left substructure $P_{\mathrm{L}}=\left\{A_{1}, B_{1}\right\}^{8}$, (b) the right substructure $P_{\mathrm{R}}=\left\{B_{2}, A_{2}\right\}^{8}$, and (c,d) the heterostructure $\mathrm{SH}=P_{\mathrm{L}} U P_{\mathrm{R}}$. Here the design wavelength for $P_{\mathrm{L}}$ and $P_{\mathrm{R}}$ are $\lambda_{\mathrm{L}}=1.45 \mu \mathrm{m}$ and $\lambda_{\mathrm{R}}=1.65 \mu \mathrm{m}$, respectively.

In order to investigate the MO responses of the considered structures, we have calculated the transmittance and FR spectra of the $\mathrm{SH}$ with optimum repetition number of $m=8$, in the wavelength range of $1-2.2 \mu \mathrm{m}$. These spectra for the separated substructures $P_{\mathrm{L}}$ and $P_{\mathrm{R}}$ are shown in Fig. 2a and b, respectively. For substructure $P_{\mathrm{L}}$ the PBG is set between $1.25 \mu \mathrm{m}$ and $1.7 \mu \mathrm{m}$ in which the design wavelength of $1.45 \mu \mathrm{m}$ is located at the PBG. While the PBG of the substructure $P_{\mathrm{R}}$ is between $1.43 \mu \mathrm{m}$ and $1.94 \mu \mathrm{m}$, which includes the design wavelength of $1.65 \mu \mathrm{m}$. For both substructures, the magnitude of FR has local maxima at the PBG edges that corresponded to the maxima in the transmittance spec- tra. The maximum angle of the FR for each substructure is at the long-wavelength edge of the PBG, which is a clear manifestation of the optical Borrmann effect in the artificially stratified media [23].

Figure 2c shows the transmittance spectrum of the heterostructure SH. The PBG of the SH covers the wavelength from $1.25 \mu \mathrm{m}$ to $1.94 \mu \mathrm{m}$, which is wider than the PBG of substructures $P_{\mathrm{L}}$ and $P_{\mathrm{R}}$. Indeed, the PBG of SH is made through overlapping the PBGs of substructures $P_{\mathrm{L}}$ and $P_{\mathrm{R}}$, that is, the short-wavelength edge of heterostructure $\mathrm{PBG}$ is the first edge of the PBG of $P_{\mathrm{L}}$, while the long-wavelength edge of heterostructure $\mathrm{PBG}$ is the end edge of the PBG of $P_{\mathrm{R}}$. The resonance transmittance of $T=0.79$ appears at $\lambda_{1}=\left(\lambda_{\mathrm{L}}+\lambda_{\mathrm{R}}\right) / 2=1.55 \mu \mathrm{m}$.

Figure $2 \mathrm{~d}$ depicts the FR spectrum of the heterostructure SH. As can be seen, a sharp peak of FR magnitude $\left(\Theta_{\mathrm{F}}=-27.35 \mathrm{deg}\right)$ occurs at the resonance wavelength of heterostructure. However, it is worthy to note that there is not any resonant transmittance and enhanced FR for design wavelengths of $\lambda_{\mathrm{L}}=1.45 \mu \mathrm{m}$ and $\lambda_{\mathrm{R}}=1.65 \mu \mathrm{m}$. While for symmetric microcavity structures the resonance transmittance with enhanced FR does occur at design wavelength [16], whereas for the magnetophotonic heterostructure, transmittance and enhanced FR would appear at the resonance wavelength of the heterostructure, instead of design wavelengths of the left or right substructures.

\subsection{A multiple magnetophotonic heterostructure}

To continue the study, we are interested in investigation of the MO responses of multiple heterostructures consisting $\mathrm{SiO}_{2} / \mathrm{Ce}: \mathrm{YIG}$ bilayers as

$$
\begin{aligned}
& \mathrm{MH}=P_{1} U P_{2} U P_{3} U P_{4}=\left\{A_{1}, B_{1}\right\}^{m_{1}}\left\{B_{2}, A_{2}\right\}^{m_{2}} \\
& \quad \times\left\{A_{3}, B_{3}\right\}^{m_{3}}\left\{B_{4}, A_{4}\right\}^{m_{4}} .
\end{aligned}
$$

In general, such a heterostructure consists of four periodic structures where each periodic structure $P_{\ell}$ is characterized by the design wavelength of $\lambda^{(\ell)}$ and the repetition number of $m_{\ell}$. To obtain a symmetric heterostructure, we suppose that all periodic structures have identical optimum repetition number of $m=7$. The general multiple heterostructure would show distinct MO responses with respect to the different design wavelengths. Hence we have categorized the derived heterostructures of $\mathrm{MH}$ as follows:

1) $\mathrm{MH}_{1}=\mathrm{MH}$, in which

$$
\lambda^{(1)}=\lambda^{(2)}=1.3 \mu \mathrm{m}, \quad \lambda^{(3)}=\lambda^{(4)}=1.8 \mu \mathrm{m},
$$

2) $\mathrm{MH}_{2}=\mathrm{MH}$, in which

$$
\lambda^{(1)}=\lambda^{(4)}=1.3 \mu \mathrm{m}, \quad \lambda^{(2)}=\lambda^{(3)}=1.8 \mu \mathrm{m},
$$

3) $\mathrm{MH}_{3}=\mathrm{MH}$, in which

$$
\lambda^{(1)}=\lambda^{(4)}=1.8 \mu \mathrm{m}, \quad \lambda^{(2)}=\lambda^{(3)}=1.3 \mu \mathrm{m},
$$

4) $\mathrm{MH}_{4}=\mathrm{MH}$, in which

$$
\lambda^{(1)}=\lambda^{(3)}=1.3 \mu \mathrm{m}, \quad \lambda^{(2)}=\lambda^{(4)}=1.8 \mu \mathrm{m} .
$$

The heterostructure $\mathrm{MH}_{1}$ can be considered as the union 
of the two symmetric microcavity structures as

$$
\begin{gathered}
\mathrm{MH}_{1}=\mathrm{MS}_{1} \cup \mathrm{MS}_{3}=\left\{A_{1}, B_{1}\right\}^{7}\left\{B_{1}, A_{1}\right\}^{7} \\
\cup\left\{A_{3}, B_{3}\right\}^{7}\left\{B_{3}, A_{3}\right\}^{7},
\end{gathered}
$$

with the design wavelength of $\lambda^{(1)}=1.3 \mu \mathrm{m}$ for left microcavity structure and $\lambda^{(3)}=1.8 \mu \mathrm{m}$ for the right one. According to Eq. (14), it can be realized that for these microcavity structures the resonance wavelengths are equivalent with the corresponding design wavelengths.

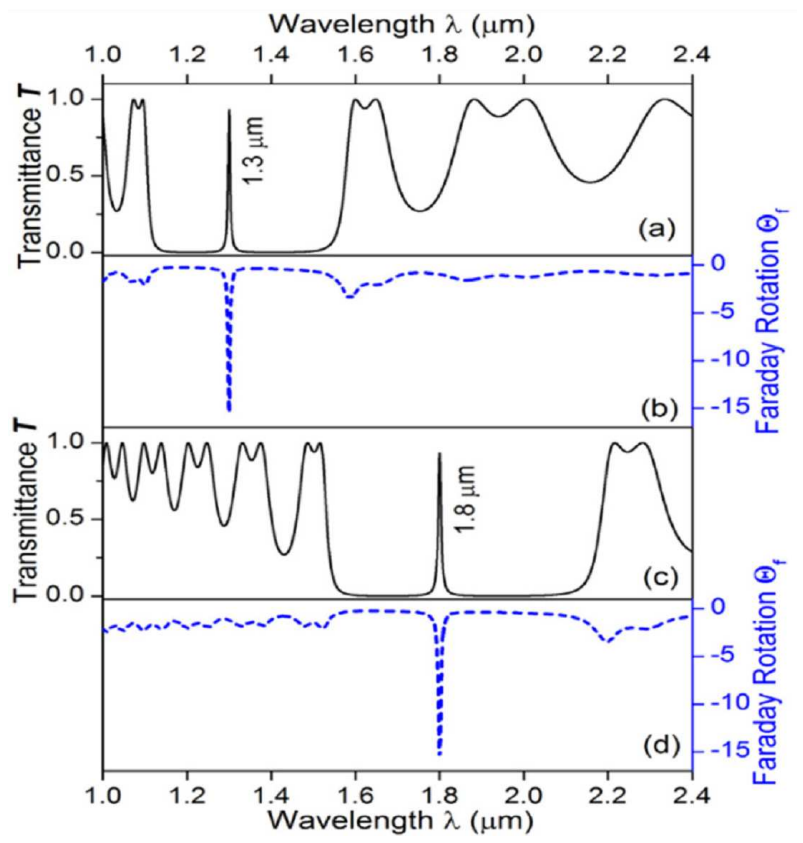

Fig. 3. Transmittance and Faraday rotation spectra for the $\mathrm{SiO}_{2} / \mathrm{Ce}: \mathrm{YIG}$ multilayers with the structure as $(\mathrm{a}, \mathrm{b})$ the microcavity structure $\mathrm{MS}_{1}=$ $\left\{A_{1}, B_{1}\right\}^{7}\left\{B_{1}, A_{1}\right\}^{7}$, (c, d) the microcavity structure $\mathrm{MS}_{2}=\left\{A_{2}, B_{2}\right\}^{7}\left\{B_{2}, A_{2}\right\}^{7}$. Here the design wavelength for $\mathrm{MS}_{1}$ and $\mathrm{MS}_{2}$ are $\lambda^{(1)}=1.3 \mu \mathrm{m}$ and $\lambda^{(2)}=$ $1.8 \mu \mathrm{m}$, respectively.

The transmittance and FR spectra of the separated microcavity structures are represented in Fig. 3. As can be seen from the transmittance spectra in Fig. 3a, the PBG of $\mathrm{MS}_{1}$ is located between $1.1 \mu \mathrm{m}$ and $1.58 \mu \mathrm{m}$ with a resonance transmittance at $1.3 \mu \mathrm{m}$. For $\mathrm{MS}_{3}$ (Fig. 3c) the PBG is laid from $1.54 \mu \mathrm{m}$ to $2.14 \mu \mathrm{m}$ and the resonance transmittance is at $1.8 \mu \mathrm{m}$. Looking the FR spectra, the enhanced FRs at resonance modes are seen for $\mathrm{MS}_{1}$ and $\mathrm{MS}_{3}$. These features of the microcavity structures was expected as reported in the number of former research works $[15,16]$.

Figure 4 shows the transmittance and FR spectra of the $\mathrm{MH}_{1}$. The wide $\mathrm{PBG}$ with about $1 \mu \mathrm{m}$ width is constructed from $1.1 \mu \mathrm{m}$ to $2.14 \mu \mathrm{m}$ with three resonance wavelengths at $1.3 \mu \mathrm{m}, 1.55 \mu \mathrm{m}$, and $1.8 \mu \mathrm{m}$, which are associated with the sharp peaks of FR magnitudes. These corner resonance modes come from the left and right microcavity structures resonance modes, while the central one is related to the heterostructure resonance

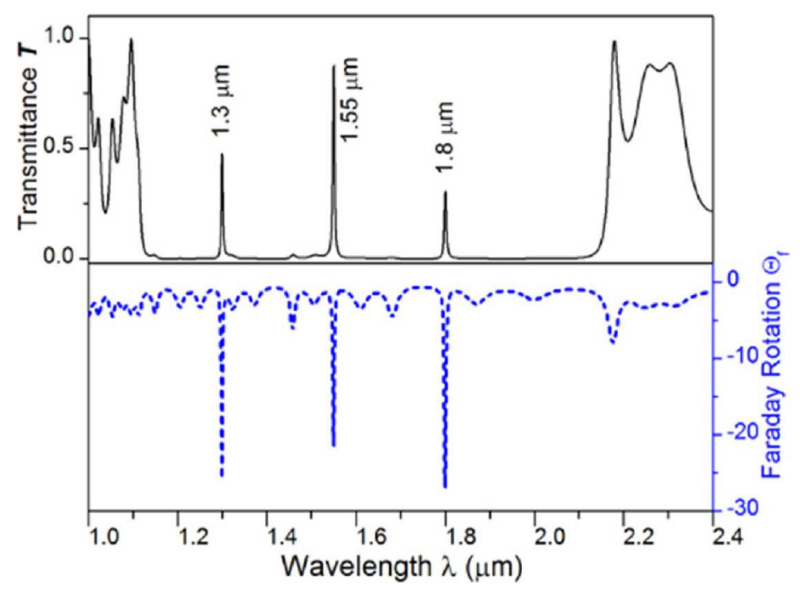

Fig. 4. Transmittance and Faraday rotation spectra for heterostructure $\mathrm{MH}_{1}=\mathrm{MS}_{1} U \mathrm{MS}_{2}$. Here the $\mathrm{MS}_{1}$ and $\mathrm{MS}_{2}$ are the same as in Fig. 3.

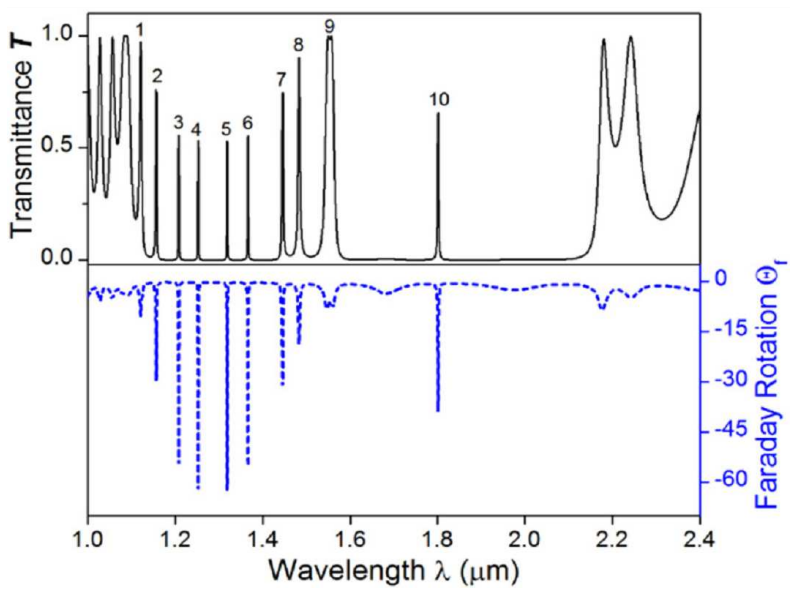

Fig. 5. Transmittance and Faraday rotation spectra for heterostructure $\mathrm{MH}_{2}=$ $\left\{A_{1}, B_{1}\right\}^{7}\left\{B_{2}, A_{2}\right\}^{7}\left\{A_{3}, B_{3}\right\}^{7}\left\{B_{4}, A_{4}\right\}^{7}$. Here the design wavelengths are $\lambda^{(1)}=\lambda^{(4)}=1.3 \mu \mathrm{m}$, $\lambda^{(2)}=\lambda^{(3)}=1.8 \mu \mathrm{m}$. The resonance transmittances are realized at (1) $1.121 \mu \mathrm{m}$, (2) $1.156 \mu \mathrm{m}$, (3) $1.209 \mu \mathrm{m}$, (4) $1.253 \mu \mathrm{m}$, (5) $1.318 \mu \mathrm{m}$, (6) $1.367 \mu \mathrm{m}$, (7) $1.445 \mu \mathrm{m}$, (8) $1.483 \mu \mathrm{m}$, (9) $1.55 \mu \mathrm{m}$ and (10) $1.8 \mu \mathrm{m}$ wavelengths.

mode described by Eq. (14). Comparing these transmittance modes and their corresponding FRs, it can be said that the highest transmittance is associated with the lowest FR and the lowest transmittance is associated with the highest FR. In other words, the trade-off relationship between the transmittance and FR of resonance modes are clearly manifested. The wavelengths $1.3 \mu \mathrm{m}$ and $1.55 \mu \mathrm{m}$ are currently used in telecommunication systems. So that, such a heterostructure would have the potential applications in designing the multichannel enhanced Faraday rotators to use in telecommunication miniaturized devices.

The transmittance and FR spectra of the heterostructures $\mathrm{MH}_{2}, \mathrm{MH}_{3}$, and $\mathrm{MH}_{4}$ are shown in Figs. 5-7, respectively. The PBG of all these heterostructures takes 


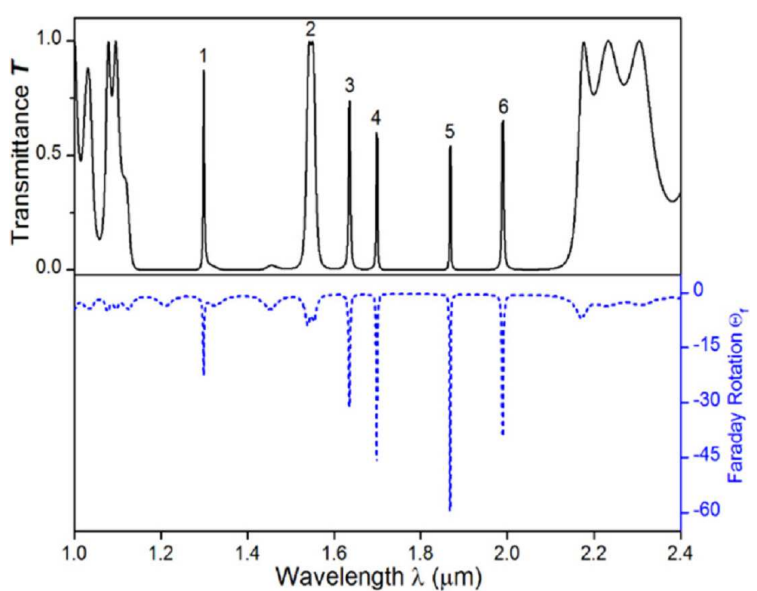

Fig. 6. Transmittance and Faraday rotation spectra for heterostructure $\mathrm{MH}_{3}=$ $\left\{A_{1}, B_{1}\right\}^{7}\left\{B_{2}, A_{2}\right\}^{7}\left\{A_{3}, B_{3}\right\}^{7}\left\{B_{4}, A_{4}\right\}^{7}$. Here the design wavelengths are $\lambda^{(1)}=\lambda^{(4)}=1.8 \mu \mathrm{m}$, $\lambda^{(2)}=\lambda^{(3)}=1.3 \mu \mathrm{m}$. The resonance transmittances are realized at (1) $1.3 \mu \mathrm{m}$, (2) $1.55 \mu \mathrm{m}$, (3) $1.636 \mu \mathrm{m}$, (4) $1.698 \mu \mathrm{m}, \quad$ (5) $1.868 \mu \mathrm{m}$, and (6) $1.989 \mu \mathrm{m}$ wavelengths.

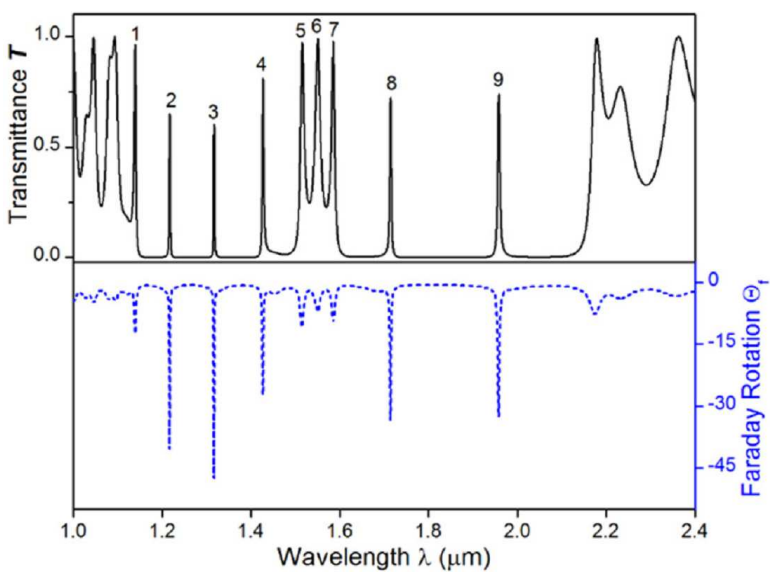

Fig. 7. Transmittance and Faraday rotation spectra for heterostructure $\mathrm{MH}_{4}=$ $\left\{A_{1}, B_{1}\right\}^{7}\left\{B_{2}, A_{2}\right\}^{7}\left\{A_{3}, B_{3}\right\}^{7}\left\{B_{4}, A_{4}\right\}^{7}$. Here the design wavelengths are $\lambda^{(1)}=\lambda^{(3)}=1.3 \mu \mathrm{m}$, $\lambda^{(2)}=\lambda^{(4)}=1.8 \mu \mathrm{m}$. The resonance transmittances are realized at (1) $1.139 \mu \mathrm{m}$, (2) $1.217 \mu \mathrm{m}$, (3) $1.317 \mu \mathrm{m}$, (4) $1.427 \mu \mathrm{m}$, (5) $1.515 \mu \mathrm{m}$, (6) $1.55 \mu \mathrm{m},(7) 1.585 \mu \mathrm{m}$, (8) $1.714 \mu \mathrm{m}$ and (9) $1.958 \mu \mathrm{m}$ wavelengths.

place between $1.1 \mu \mathrm{m}$ and $2.14 \mu \mathrm{m}$, the same as the PBG of the heterostructure $\mathrm{MH}_{1}$. Consequently, it can be revealed that the BPG of the general multiple heterostructure $\mathrm{MH}$ is independent of the substructures design wavelengths.

For $\mathrm{MH}_{2}$, the $P_{2} U P_{3}$ can be considered as the microcavity structure with a central dielectric layer and the design wavelength of $1.8 \mu \mathrm{m}$, which is surrounded by two mirror symmetric periodic structures of $P_{1}$ from left hand side and $P_{4}$ from right hand side, where $P_{1}$ and $P_{4}$ have the identical design wavelengths of $1.3 \mu \mathrm{m}$. Such a consideration can be made for the $\mathrm{MH}_{3}$ only by exchanging the design wavelengths of central microcavity structure and the corner periodic structures.

Looking the transmittance spectra of the heterostructures $\mathrm{MH}_{2}, \mathrm{MH}_{3}$, and $\mathrm{MH}_{4}$, we see that there is a resonant transmittance at the $1.55 \mu \mathrm{m}$, but the width of this mode for $\mathrm{MH}_{2}$ and $\mathrm{MH}_{3}$ is wider in comparison with $\mathrm{MH}_{4}$ one. To illustrate the spectral properties of these heterostructures it is better to split their PBG into left and right parts, regarding the central resonance mode of $1.55 \mu \mathrm{m}$. For heterostructure $\mathrm{MH}_{2}$ there is only one resonance mode in the right part of PBG at the $1.8 \mu \mathrm{m}$, while there are eight resonance modes at $1.121,1.156,1.209$, $1.253,1.318,1.367,1.445$, and $1.483 \mu \mathrm{m}$ wavelengths in the left part of the PBG. In contrary, the left part of PBG of the $\mathrm{MH}_{3}$ has only one resonance modes at the $1.3 \mu \mathrm{m}$, while there are four resonances at 1.636, 1.698, 1.868 and $1.989 \mu \mathrm{m}$ wavelengths in the right part. The enhanced FRs occur at all of resonance wavelengths and their FR magnitudes are larger for lower transmittances. So that the highest transmittance of these two heterostructures which realized at $1.55 \mu \mathrm{m}$ show the lowest FRs.

The PBG of the heterostructure $\mathrm{MH}_{4}$ includes five resonances at $1.139,1.217,1.317,1.427$, and $1.515 \mu \mathrm{m}$ wavelengths in the left part and three resonances at 1.585 , 1.714 , and $1.958 \mu \mathrm{m}$ wavelengths in the right part of the PBG. The width of the central $1.55 \mu \mathrm{m}$ mode is not as wide as the $\mathrm{MH}_{2}$ and $\mathrm{MH}_{3}$ ones, nevertheless, for $\mathrm{MH}_{4}$ there are two resonance transmittances in the vicinity of the central mode. Similar to aforementioned cases, the sharp peaks of the FR magnitudes are associated with all resonance modes of $\mathrm{MH}_{4}$ and the transmittances and FRs of these modes follow the trade-off relationship.

\section{Conclusion}

The multichannel enhanced FRs resulting from resonance transmittances of magnetophotonic heterostructures are demonstrated. For a simple magnetophotonic heterostructure, it is shown that the high transmittance enhanced FR in a wide PBG can be adjusted to the desired wavelength through introducing special substructures with optimized repetition numbers and proper layer thicknesses. Also, the transmittance MO responses of different configurations of a multiple magnetophotonic heterostructure are investigated. It is shown that each configuration has unique features of multichanneled enhanced FRs. Analyzing the results, it is found that the multiple magnetophotonic heterostructure constituting of two microcavity substructures with design wavelengths of $1.3 \mu \mathrm{m}$ and $1.8 \mu \mathrm{m}$ could support both telecommunications resonance wavelengths of $1.3 \mu \mathrm{m}$ and $1.55 \mu \mathrm{m}$ simultaneously. We expect that the obtained results have potential applications in designing the multi-function single magneto-optical devices such as multiple Faraday rotators and wavelength division multiplexing systems. 


\section{References}

[1] J.D. Joannopoulos, S.G. Johnson, J.N. Winn, R.D. Meade, Photonic Crystals: Modeling the Flow of Light, 2nd ed., Princeton University Press, Princeton (NJ) 2008.

[2] P. Markos, C.M. Soukoulis, Wave Propagation: From Electrons to Photonic Crystals and Left-Handed Materials, Princeton University Press, Princeton (NJ) 2008.

[3] E. Yablonovitch, Phys. Rev. Lett. 58, 2059 (1987).

[4] S. John, Phys. Rev. Lett. 58, 2486 (1987).

[5] D.R. Smith, R. Dalichaouch, N. Kroll, S. Schultz, S.L. McCall, P.M. Platzman, J. Opt. Soc. Am. B 10, 314 (1993).

[6] A. Rudziński, Acta Phys. Pol. A 111, 323 (2007).

[7] M. Kohmoto, B. Sutherland, K. Iguchi, Phys. Rev. Lett. 58, 2436 (1987).

[8] M. Dulea, M. Severin, R. Riklund, Phys. Rev. B 42 3680 (1990).

[9] W.J. Hsueh, S.J. Wun, Z.J. Lin, Y.H. Cheng, J. Opt. Soc. Am. B 28, 2584 (2011).

[10] L. Wang, Z. Wang, Y. Wu, L. Chen, S. Wang, X. Chen, W. Lu, J. Appl. Phys. 95, 424 (2004).

[11] P. Han, H. Wang, J. Opt. Soc. Am. B 22, 1571 (2005).

[12] W. Zhang, J. Liu, W.P. Huang, W. Zhao, J. Opt. Soc. Am. B 26, 1845 (2009).
[13] N. Ansari, M.M. Tehranchi, Acta Phys. Pol. A 115, 378 (2009)

[14] M. Levy, H.C. Yang, M.J. Steel, J. Fujita, J. Lightwave Technol. 19, 1964 (2001).

[15] H. Kato, T. Matsushita, A. Takayama, M. Egawa, K. Nishimura, M. Inoue, J. Appl. Phys. 93, 3906 (2003).

[16] M. Inoue, R. Fujikawa, A. Baryshev, A. Khanikaev, P.B. Lim, H. Uchida, O. Aktsipetrov, A. Fedyanin, T. Murzina, A. Granovsky, J. Phys. D, Appl. Phys. 39, R151 (2006).

[17] M. Zamani, M. Ghanaatshoar, H. Alisafaee, J. Opt. Soc. Am. B 28, 2637 (2011).

[18] M.J. Steel, M. Levy, R.M. Osgood, IEEE Photon. Technol. Lett. 12, 1171 (2000).

[19] T. Sun, J. Luo, P. Xu, L. Gao, Phys. Lett. A 375, 2185 (2011)

[20] N. Ansari, S.I. Khartsev, A.M. Grishin, Opt. Lett. 37, 3552 (2012).

[21] Š. Višn̆ovský, K. Postava, T. Yamaguchi, Czech. J. Phys. 51, 917 (2001).

[22] R.M.A. Azzam, N.M. Bashara, Ellipsometry and Polarized Light, North Holland, Amsterdam 1988.

[23] A.B. Khanikaev, A.B. Baryshev, P.B. Lim, H. Uchida, M. Inoue, A.G. Zhdanov, A.A. Fedyanin, A.I. Maydykovskiy, O.A. Aktsipetrov, Phys. Rev. B 78, 193102 (2008). 